\title{
Through Silicon Via-Based Grid for Thermal Control in 3D Chips
}

\author{
José L. Ayala ${ }^{1}$, Arvind Sridhar ${ }^{2}$, Vinod Pangracious ${ }^{2}$, David Atienza ${ }^{2}$, and \\ Yusuf Leblebici ${ }^{3 \star}$ \\ 1 Dept. of Computer Architecture and Systems Engineering (DACYA) \\ Complutense University of Madrid, Spain. \\ E-mail: jayala@fdi.ucm.es \\ 2 Embedded Systems Laboratory (ESL), EPFL, Switzerland. \\ E-mail: \{arvind.sridhar, vinod.pangracious, david.atienza\} @epfl.ch \\ 3 Microelectronics Systems Laboratory (LSM), EPFL, Switzerland. \\ E-mail:yusuf.leblebici@epfl.ch
}

\begin{abstract}
D stacked chips have become a promising integration technology for modern systems. The complexity reached in multi-processor systems has increased the communication delays between processing cores, and an effective way to diminish this impact on communication is the $3 \mathrm{D}$ integration technology and the use of through-silicon vias (TSVs) for inter-layer communication. However, 3D chips present important ther- mal issues due to the presence of processing units with a high power density, which are not homogeneously distributed in the stack. Also, the presence of hot-spots creates thermal gradients that impact negatively on the system reliability and relate with the leakage power consumption. Thus, new approaches for thermal control of $3 \mathrm{D}$ chips are in great need. This paper discusses the use of a grid and non-uniform placement of TSVs as an effective mechanism for thermal balancing and control in 3D chips. We have modelled the material layers and TSVs mathematically using a detailed calibration phase based on a real 5-tier 3D chip stack, where several heaters and sensors are manufactured to study the heat diffusion. The obtained results show interesting conclusions about thermal dissipation for $3 \mathrm{D}$ chips with TSVs and outline new insights in the area of thermal modeling and optimization for 3D chips by exploiting the inclusion of minimal percentages of TSVs in strategic positions of the layout.
\end{abstract}

\section{Introduction}

Three-dimensional (3D) integration consists of vertical placement and interconnect of several layers of active circuits. The main interests of this technology are the reduciton of global interconnection lengths, the increase circuit functionality and enabling new compact circuit architectures [1-3].

A key component of 3D technology is the through-silicon via (TSV), which enables communication between two dies as well as with the global package. Several works have addressed the layout optimization of lacement of vias for heat dissipation in

\footnotetext{
* This work was partly supported by the Swiss Confederation through the Nano-Tera.ch NTF Project nr. 123618 - CMOSAIC, and the Spanish Government Research Grant TIN200800508 .
} 
3D ICs $[4,5]$. Other works [6] propose analytical and finite-element models of heat transfer in 3D electronic circuits and use these models to analyze the impact of various geometric parameters and thermophysical properties (through silicon vias, inter-die bonding layers, etc.) on thermal performance of a 3D IC.

To the best of our knowledge, this is the first work that proposes the use of a nano-structure grid of TSVs to effectively optimize the thermal profile in 3D stacks. The closest work to our proposal is [7], where the authors analyze the impact of thermal through silicon vias (TTVs) in vertically integrated die-stacked devices. However, while the work presented in [7] performs a theoretical analysis, our approach proposes an accurate thermal modeling of the TSVs and it is validated against measurements collected in a real chip. Finally, the thermal effect of the nano-structure of the TSVs is carefully examined.

The experimental work of this paper is carried out through a novel thermal analysis of a real 5-tier 3D stack (see Figure 11). Then, the material layers and TSVs are modeled mathematically, and the effect of a non-homogeneous distribution of the vias for thermal control is analyzed and effective inclusion of localized TSVs conforming a grid of nanostructures for thermal control is proposed. Also, the effect of specific interface materials used as inter-layer glue is considered. These interfaces expose unique characteristics due to the presence of aluminium dopants, which is another tested solution for a more effective thermal distribution in forthcoming $3 \mathrm{D}$ chips.

The paper structure is as follows: Section 2 presents the configuration of the 3D stack developed for the experimental work, and the developed thermal model is explained in Section 3. Then, the experimental work is covered in Section 4 Finally, in Section 5 we summarize the main conclusions of this work.

\section{Configuration of 3D Stack for Thermal Analysis}

The 3D chip manufactured for our experimental setup is created as a multi-level chip, built by stacking silicon layers and fixed with an interface glue. In this configuration, we can find five silicon layers (Die 1 - Die 5), the epoxy-based interface glue, and a bottom PCB layer (see Figure 1). Each stack has an area of $1 \mathrm{~cm}^{2}$.
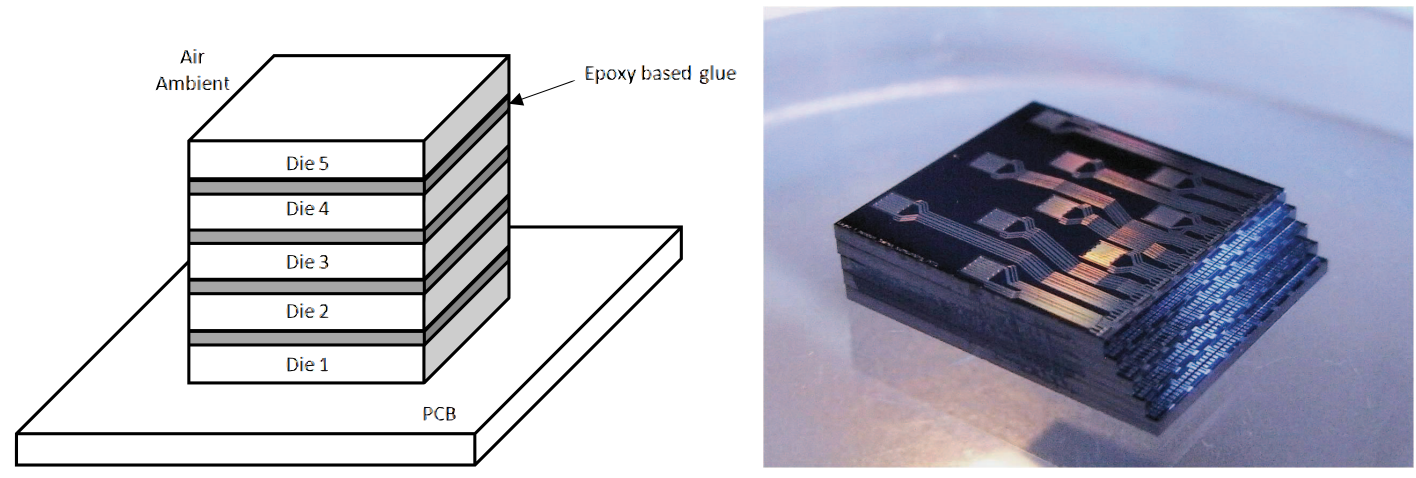

Fig. 1. Test 3D stacked structure (left - representation model ; right - 3D chip). 
This 3D stack resembles the thermal effects that can be found in a 3D multiprocessor systems on chip by the use of heaters that create the power dissipation. As the power dissipated in a chip is not uniform on its surface (microprocessors can dissipate between 200 to $300 \mathrm{~W} / \mathrm{cm}^{2}$ while memories only dissipate about $10 \mathrm{~W} / \mathrm{cm}^{2}[2,3]$ ) each layer contains several microheaters located at different points to simulate the heat dissipated by the integrated components.

These microheaters are built as a serpentine wire created with thin-film technologies. The material used for the heaters is Platinum, due to its capability to operate at very high temperature and its long stability. Within each die, these aluminum resistorbased heaters are fabricated in the silicon dioxide layer on the top of the substrate. Hence, their placement and thermal effects are effectively modeling the hot-spot cores in an actual 3D multi-processor system on chip (MPSoC) architecture.

Several thermal sensors are also placed in specific places as detector devices to monitor the temperature inside of the stack and check the heat dissipated and the heat interactions between neighboring microheaters. Platinum has also been selected as the material to build the sensors; therefore, sensors and microheaters can be manufactured at the same time in a single step of the technology process. These sensors are resistance temperature detectors (RTDs). In this way, the temperature of the heater creates a variation in the resistance of the sensor. Then, the temperature can be obtained by the observation of the voltage drop at both extremities of the sensor (with a fixed current) and applying the resistivity temperature dependence of Platinum.

Each layer comprises 10 heaters of $1 \mathrm{~mm}^{2}$ each, very similar to the area of common processing elements. These microheaters have been designed to resemble a hot-spot on the surface of the chip of $300 \mathrm{~W} / \mathrm{cm}^{2}$; therefore, each heater can dissipates 1-9W. The heaters are aligned in three vertical lines. The 5 layers of the stack have the same configuration so the alignment of the heaters appears also out of the plane.

In our configuration, RTDs are placed around the heaters. These sensors are designed for a value of $100 \Omega$ and are driven with a current of $1 \mathrm{~mA}$.
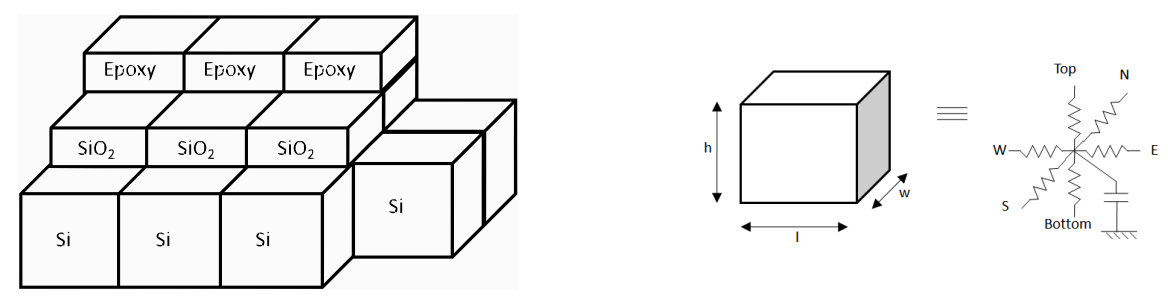

Fig. 2. (a) The unitary thermal cells of the 3D stack. (b) Equivalent RC circuit of a single cell.

\section{3D Stack Thermal Model}

In this section we present the thermal model we have developed for the five-layered 3D stack structure considered in this work (cf. Figure 1). As shown in this figure, five silicon dies, stacked one on the top of another fixed with an interface epoxy glue, are placed on the printed circuit board (PCB). The bottom surface of the 3D stack attached to the PCB is assumed to be adiabatic; therefore, the heat will be exchanged through the vertical active and interface layers in the system. 
Table 1. Thermal properties of materials.

\begin{tabular}{|c|c|}
\hline Silicon thermal conductivity & $295-0.491 \mathrm{~T} \mathrm{~W} / \mathrm{mK}$ \\
\hline Silicon specific heat & $1.659 \times 10^{6} \mathrm{~J} / \mathrm{m}^{3} \mathrm{~K}$ \\
\hline $\mathrm{SiO}_{2}$ thermal conductivity & $1.38 \mathrm{~W} / \mathrm{mK}$ \\
\hline $\mathrm{SiO}_{2}$ specific heat & $4.180 \times 10^{6} \mathrm{~J} / \mathrm{m}^{3} \mathrm{~K}$ \\
\hline Aluminum electrical resistivity & $2.82 \times 10^{-8}(1+0.0039 \Delta T) \Omega m$ \\
& $\Delta T=T-293.15 \mathrm{~K}$ \\
\hline
\end{tabular}

Due to 3D stack structure, the heat generated by the heat sources must flow through the body of the 3D tiers, and end at the environment interface (ambient) where it is spread through natural convection. Then, the heat flow inside this structure is diffusive in nature and hence, is modeled by its equivalence to an electronic RC circuit [8-10]. This is done by first dividing the entire structure into small cubical thermal cells as shown in Figure $2 k$. Each cell is then modeled as a node containing six resistances that represent the conduction of heat in all the six directions (top, bottom, north, south, east and west), and a capacitance that represents the heat storage inside the cell, as shown in Figure 20].

Current sources, representing the sources of heat, are connected to the cells in the regions where the Aluminum heaters are present. The entire circuit is grounded to the ambient temperature at the top and the side boundaries of the 3D stack through resistances, which represent the thermal resistance from the chip to the air ambient.

The behavior of the resulting resistance-capacitance (RC) circuit can be described using a set of first order differential equations via nodal analysis [11].

This model considers the temperature-dependent thermal conductivity of silicon and the temperature-dependent electrical resistance of the aluminum heaters respectively. In this work, a first-order dependence of these parameters on temperatures around $300 \mathrm{~K}$ is assumed. They key thermal parameters of the five-tier 3D stack are shown in Table 1012].

Finally, for the validation of the proposed 3D thermal library, extensive temperature measurements, combining multiple heaters of the ten available in each of the five tiers on the 3D stack, were performed with DC current inputs for the heaters (covering the aforementioned $1 \mathrm{~W}$ to $9 \mathrm{~W}$ per heater), where differences of less than $1 \%$ in intra-tier thermal distribution, and less than $2 \%$ in inter-tier thermal distribution, in the 5 -tier 3D chip were measured.

\section{TSVs Thermal Characterization and Experimental Balancing}

In the last years, many fabrication-based solutions for the thermal management in 3D integrated circuits have been proposed. Thermal through silicon vias (TTSVs) [6] are a very promising principle. TTSVs are based on the principle that it is more desirable to reduce the difference in the temperatures between various parts of the IC, rather than the reduction of the absolute temperature of the chip. Indeed, variations in operating temperatures affect performance of different parts of the IC (e.g. processor and memory) differently, leading to timing errors and undesirable irregular chip failures. 

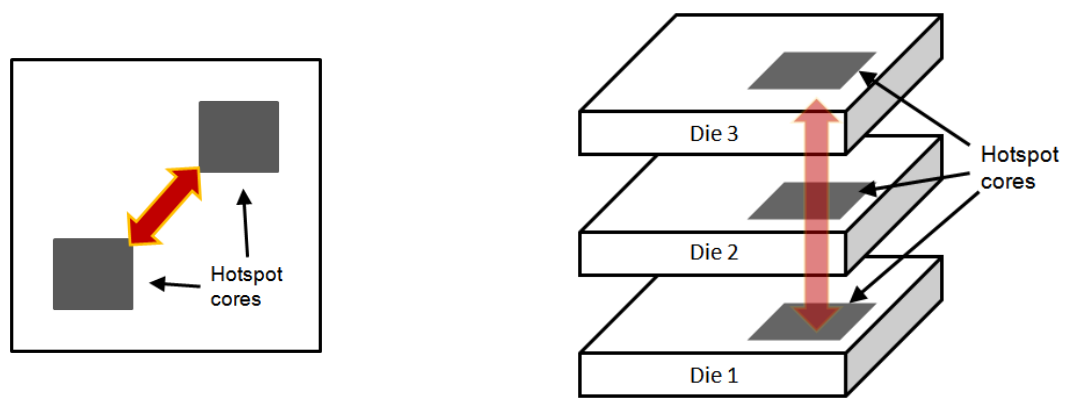

Fig. 3. Communication between active cores in a 3D IC (a)within one layer (b)between different layers.
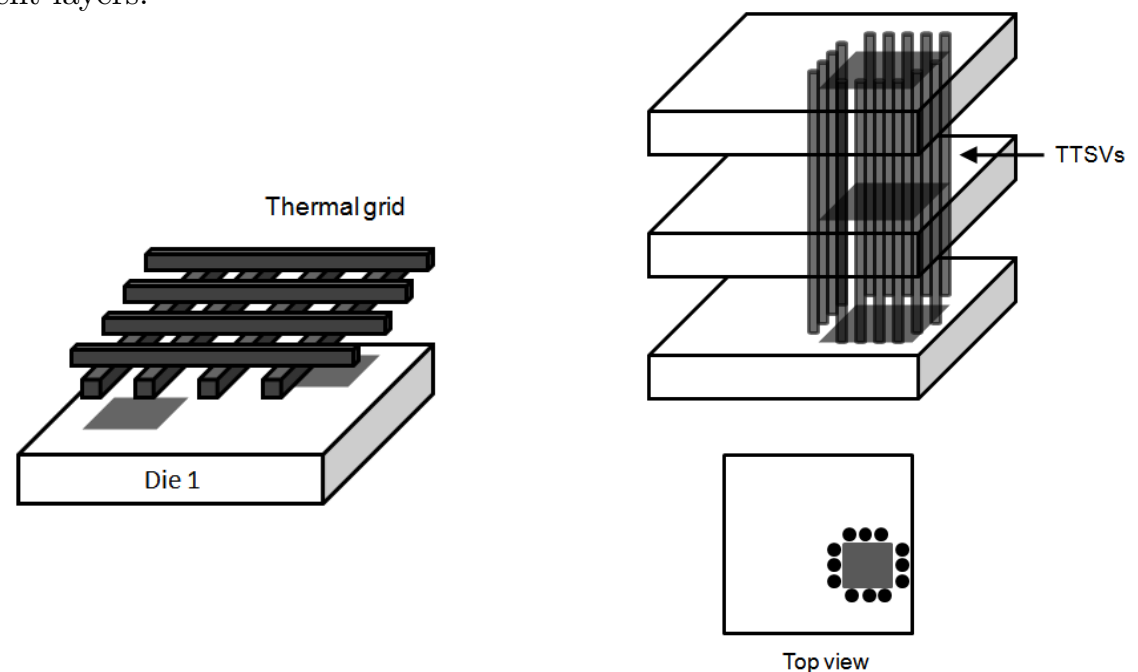

Fig. 4. (a) Thermal grid for reducing temperature variation within a single layer. (b) TTSVs for reducing temperature variations along the different layers in a 3D IC.

Moreover, thermal gradients have been observed as a determinant negative factor on system reliability.

To overcome the aforementioned challenges in thermal management and to simulate the effects of on-chip metallizations on the thermal behavior of the 3D stack, TTSVs and thermal grids were introduced in the thermal model developed in the previous section. For a complete fine-grained thermal exploration and to avoid the influence of direct contact to air of the top and bottom tiers, a 3-layered 3D stack was used without instead of the 5-layered stack. Figure 3 shows two test cases- (a) with two hot-spot cores in the same die of the 3D stack and (b) with three cores, one on the top of another, communicating each other through different layers (from a performance perspective [3], it would be very desirable to place the most frequently communicating cores of a 3D IC one on the top of the other to reduce communication delay).

For case (a), to reduce the temperature variations within the same layer, thermal grid networks- dedicated metallizations as well as existing metallizations for the electronic design, are proposed. These thermal grid networks lower the effective thermal 


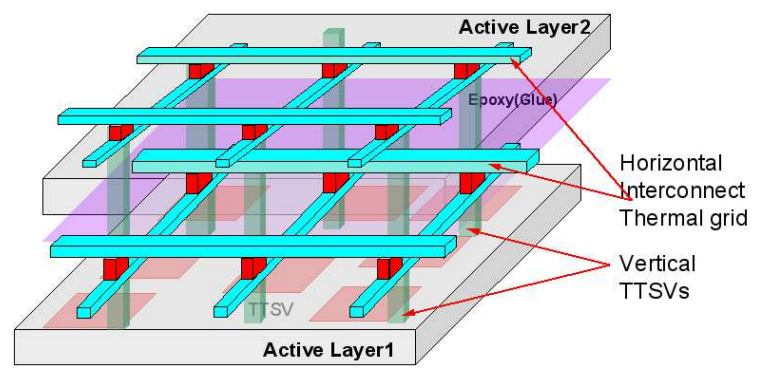

Fig. 5. Vertical and horizontal thermal grid.

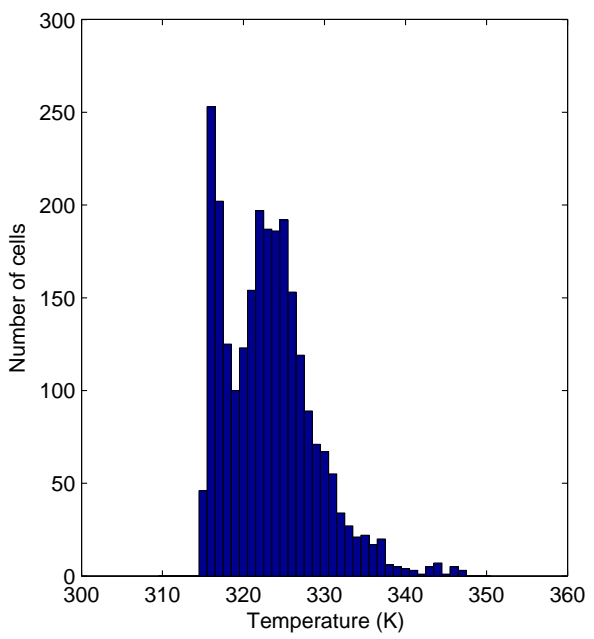

(a)

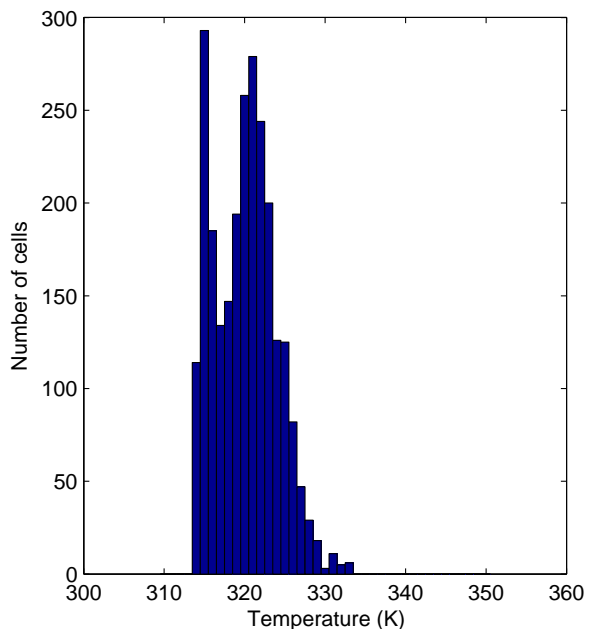

(b)

Fig. 6. Lateral temperature distribution profile for Die 1 (a)without thermal grid and (b)with thermal grid.

conductivity of the dielectric material within the layer and hence, reduce the temperature variations in the layer. This is illustrated in Figure $4(\mathrm{a})$, which shows the schematic configuration of the horizontal grid.

For case (b), to address the temperature variations between different layers in regions where the communicating cores exist, TTSVs are placed around the active cores, as shown in Figure 4(b). This placement of TTSVs, in addition to the metallizations that naturally exist between the cores for electronic routing, reduces the effective thermal conductivity of this region. This, in turn, brings the temperature of different parts of this region closer to each other because of the favored thermal flow.

To incorporate both the thermal grid and the TTSVs in the thermal model, the effective thermal conductivity was calculated for the cells in the region containing these metallizations, using the following relation: 


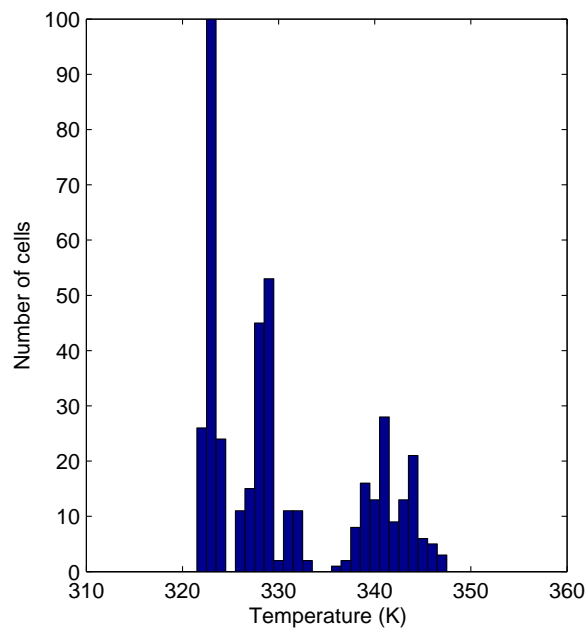

(a)

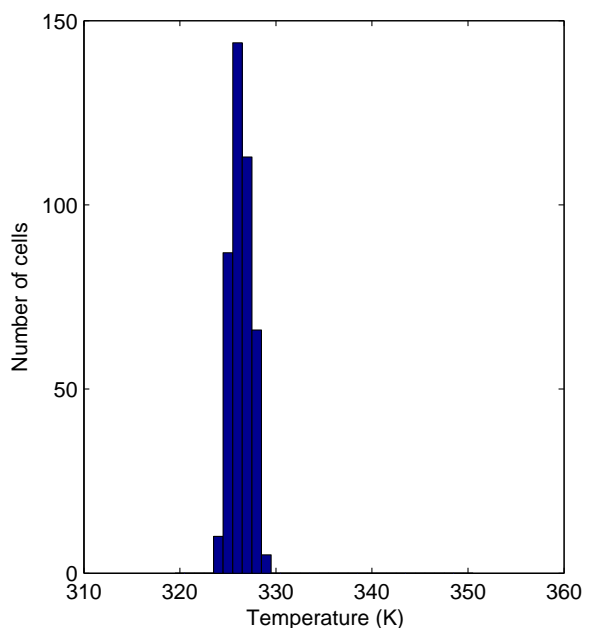

(b)

Fig. 7. Vertical temperature distribution profile for region around D06 (a)without TTSVs and (b)with TTSVs.

$$
k_{e f f}=k_{c u} \omega+k_{t h}(1-\omega),
$$

where, $k_{c u}$ is the thermal conductivity of copper (the metal used for all metallizations in the IC), $k_{t h}$ is the thermal conductivity of the surrounding material and $\omega$ is the wiring/via density in the region. In the case of TTSVs, a slight modification was made for the effective thermal conductance in the lateral direction. This parameter was calculated by computing the equivalent thermal resistance of the cells depending upon the path of heat flow while traversing it along north-south and east-west direction (a series/parallel combination of vias and surrounding material). Hence, anisotropic cells were created in order to more accurately capture the effects of TTSVs.

Figure[5]shows the devised nano-grid of horizontal interconnects and vertical TTSVs for 3D ICs. In fact, the TTSVs that integrate the nano-structure improve the overall thermal conductivity of the active layer, provided the thermal coupling is good between the TTSVs. To improve the thermal coupling, these TTSVs must be placed as close as possible to each other but electrically isolated. On the other hand, the horizontal grid helps to spread the temperature along the die and also improve the thermal conductivity of the Inter-Layer Material.

Two experiments were performed to measure the performance of these two strategies. In the first experiment, 4 heaters (in devices D02, D04, D07 and D10) in Die 1 of the 3-layered 3D stack were excited, each with a current of $300 \mathrm{~mA}\left(1.25 \mathrm{~W} / \mathrm{mm}^{2}\right)$. First, this experimental setup was simulated without any thermal grid. Next, the thermal grid was added to Die 1 (with $50 \%$ wiring density) in the same experimental setup and the resulting model was simulated again. The temperature distribution profile was drawn for each case. These histograms are shown in Figure [6 As can be seen from this 
figure, the temperature spread within this layer has been reduced by the effect of the thermal grid, that easies the diffusion of the extra heat.

In the next experiment, the same set up was used. TTSVs were laid around each of the active heaters in Die 1. The resulting thermal circuit was then simulated, once without the TTSVs and then once with the TTSVs. Temperatures in the region covered by the TTSVs of one of the heaters (the region enclosed by the TTSVs encompassing all the 3 dies) were recorded in each case. The corresponding temperature distribution profiles for one such active heater regions are shown in Figure $\mathbf{7}$ We find that the temperature spread was considerably reduced in this region along the vertical direction. Therefore, the grid of TTSVs can be considered as an effective mechanism to optimize the thermal profile in 3D stacks, both in the vertical and lateral direction.

\section{Conclusions}

This paper has proposed the use of a nano-grid of TSVs as an effective mechanism to optimize the thermal profile of 3D integrated systems. In this work, an accurate modeling of the thermal effects that appear in these structures has been developed in a 5-tier 3D stack, where a detailed thermal validation process has been performed.

Then, the proposed and tuned thermal model has been used to evaluate the capability of a nano-grid structure of thermal through-silicon vias to improve the thermal response of this complex 3D IC system. Furthermore, the nano-grid solution has been tested and configured to reduce the impact of high-density temperature hot-spots, providing very positive results in the optimization and homogeneization of the vertical and lateral diffusion of heat in all real-life working conditions. Thus, this results open a very promising new research area in the design of thermally-balanced 3D ICs.

\section{References}

1. S. Das, A. Chandrakasan, and R. Reif, "Design tools for 3-D integrated circuits," in Proc. of ASP-DAC, 2003, pp. 53-56.

2. K. Banerjee et al., "3-D ICs: a novel chip design for improving deep-submicrometer interconnect performance and systems-on-chip integration," Proceedings of the IEEE, no. 5, pp. 602-633, 2001.

3. A. W. Topol et al., "Three-dimensional integrated circuits," IBM Journal of Research and Development, no. 4-5, pp. 494-506, 2006.

4. J. Cong et al., "Thermal via planning for 3-D ics," in Proc. of ICCAD, 2005, pp. 745-752.

5. B. Goplen et al., "Placement of thermal vias in 3-d ics using various thermal objectives," IEEE T-CAD, vol. 25, no. 4, pp. 692-709, 2006.

6. A. Jain, et al., "Thermal modeling and design of 3D integrated circuits," in Proc. of Int. Conf. on TTPES, 2008.

7. V. Natarajan, et al., "Thermal and power challenges in high performance computing systems," in Proc. ISTD-TPE, 2008.

8. S. Heo, et al., "Reducing power density through activity migration," Proc. of ISPD, 2003.

9. K. Skadron, et al., "Temperature-aware microarchitecture: modeling and implementation," TACO, pp. 94-125, 2004.

10. H. Su, et al., "Full chip leakage estimation considering power supply and temperature variations," Proc. of ISPD, pp. 78-83, 2003.

11. J. Vlach et al., Computer methods for circuit analysis and design. Springer, 1983.

12. F. P. Incropera, et al., Fundamentals of heat and mass transfer. John Wiley and Sons, 2007. 